\title{
Postpartum Breastfeeding and Cardiovascular Risk Assessment in Women Following Pregnancy Complications
}

\author{
Julie Yu, BHSc, Jessica Pudwell, MPH, ${ }^{1}$ Natalie Dayan, MD, MSc, ${ }^{2}$ and Graeme N. Smith, MD, PhD, FRCSC ${ }^{1}$
}

\begin{abstract}
Background: Breastfeeding is associated with lower cardiovascular (CV) risk over the long-term, however, less is known about its immediate effects among women with a recent complicated pregnancy. The objective of this study is to investigate the short-term effects of breastfeeding on markers of cardiovascular disease risk among women $\sim 6$ months after a pregnancy complicated by a hypertensive disorder, gestational diabetes, intrauterine growth restriction, abruption, or preterm birth.

Materials and Methods: Our cross-sectional analysis includes 622 women seen at 6 months postpartum (interquartile range: 5.7-6.7) between November 2011 and December 2017 at a tertiary care center. Self-reported breastfeeding status and measured CV risk factors were assessed at the same visit. CV risk factors were compared between women who did not breastfeed $(n=100,16 \%)$, those who breastfed for less than 6 months $(n=315,51 \%)$, and those who breastfed for 6 months or more $(n=207,33 \%)$ using multivariate logistic and linear regression.

Results: Increased breastfeeding duration significantly decreased the likelihood of metabolic syndrome (adjusted odds ratio [95\% confidence interval; CI]: 0.89 [0.79-0.99]), abnormal fasting glucose (0.79 [0.64-0.96]), and ratio of total cholesterol to high-density lipoprotein-cholesterol (HDL-C) (0.86 [0.78-0.95]). Furthermore, body mass index (estimated beta coefficients $[95 \% \mathrm{CI}]-0.10[-0.18$ to -0.02$]$ ), fasting glucose $(-0.05[-0.08$ to $-0.02])$, triglycerides $(-0.07$ [ -0.10 to -0.04$])$, and ratio of total cholesterol to HDL-C ( -0.06 [ -0.10 to -0.03$])$ also decreased with increased breastfeeding duration, while HDL-C increased (0.02 [-0.01 to -0.04$])$.

Conclusions: Our findings suggest that breastfeeding is associated with decreased indicators of CV risk in a cohort of women with recent pregnancy complication.
\end{abstract}

Keywords: postpartum, breastfeeding, cardiovascular health, pregnancy complications

\section{Introduction}

C ARDIOVASCUlar DISEASE (CVD) Is the leading cause of death in women worldwide. ${ }^{1}$ Evidence suggests that pregnancy acts as a cardiovascular (CV) stress test such that the development of certain pregnancy complications (hypertensive disorders of pregnancy [HDP], gestational diabetes $[\mathrm{GDM}]$, and intrauterine growth restriction [IUGR]), identifies a woman to be at increased risk of future CVD. ${ }^{2-4}$

There is growing evidence suggesting that breastfeeding lowers specific CVD risk factors such as maternal blood pressure (BP), metabolic parameters, and risk of developing type 2 diabetes in the general population. ${ }^{5-11}$ A recent systematic review of 21 studies concluded that breastfeeding was associated with improvements in various $\mathrm{CV}$ risk factors such as hypertension, type 2 diabetes, and inflammatory markers, including ghrelin and peptide YY. ${ }^{12}$ Although there have been studies investigating the link between breastfeeding and long-term markers of CVD and morbidity in the general population, outcomes were usually measured decades after delivery, making it difficult to conclude direct associations.

There is a paucity of research on the short-term or longterm effects of breastfeeding on CVD risk specifically in women with pregnancy complications, although these women have lower initiation rates of breastfeeding and are at higher risk of early weaning. ${ }^{13-16}$ An analysis of 379 U.S. women with a history of gestational hypertension $(\mathrm{GH}) \mathrm{re}-$ ported a mean decrease of $16.3 \mathrm{mmHg}$ systolic/16.8 $\mathrm{mmHg}$ diastolic pressure in women who breastfed for more than 6 months compared with those who did not. ${ }^{17}$ Another

\footnotetext{
${ }^{1}$ Department of Obstetrics and Gynecology, Kingston Health Sciences Center, Queen's University, Kingston, Canada.

${ }^{2}$ Department of Medicine, McGill University, Montreal, Canada.
} 
prospective cohort study of 1,238 women in the United States showed a graded inverse association between breastfeeding duration and diabetes incidence. ${ }^{18}$

The postpartum period serves as a window of opportunity to implement targeted screening, counseling for lifestyle modification and potentially initiation of therapeutic interventions for $\mathrm{CV}$ health preservation and CVD prevention. ${ }^{19}$ Postpartum CV health clinics have been developed to initiate early screening, education, and intervention to reduce CVD risk in women at risk based on recent complications in pregnancy. $^{20,21}$ In this study, we analyzed data from the Maternal Health Clinic (MHC) in Kingston, Ontario, a large Canadian clinical cohort of postpartum women with recent pregnancy complications (HDP, GDM, IUGR, placental abruption, and idiopathic preterm birth), to evaluate the short-term effects of breastfeeding on a variety of CVD risk factors at 6 months postpartum.

\section{Materials and Methods}

\section{Study design, population, and setting}

This cross-sectional analysis includes all women with a recent cardiometabolic complication in pregnancy, including HDP (pre-eclampsia, HELLP syndrome, or GH), GDM, IUGR, abruption, and preterm birth, who delivered at Kingston Health Sciences Center and attended the MHC. The Kingston Health Sciences Center is a tertiary referral center in Ontario, Canada serving a multiethnic population of 500,000 people, and where over 2,000 deliveries are performed annually. All eligible women are referred at the time of delivery for a 6-month follow-up at the MHC, where they undergo physical and biochemical CVD risk screening and self-report breastfeeding status and duration. ${ }^{20}$ Women that attended the clinic from November 1, 2011 until December 31, 2017 were eligible for inclusion in this study. The Queen's University and Affiliated Teaching Hospitals Research Ethics Board reviewed the study for ethical conduct and participants provided informed written consent.

\section{Data collection procedures}

Nurses and obstetricians at the clinic collected data based on standardized interview and data collection forms and data were entered in the database by a nurse and research assistant. Blood draws were performed 1-2 weeks before the clinic visit at a community laboratory as part of local standard of care.

\section{Exposure to breastfeeding}

At the time of the MHC visit, all women were questioned by the research nurse about breastfeeding using a standardized questionnaire. Breastfeeding exposure was defined by the duration (in months) of breastfeeding at the time of the woman's MHC appointment. While MHC visits are typically scheduled for 6 months postpartum, there is some variability in the timing of this visit, and a woman is invited to attend up to 1 year postpartum.

Breastfeeding exposure was defined as the reported number of consecutive or cumulative months she had breastfed, up to and including the date of the clinic visit. Number of months of breastfeeding was recorded to one decimal point.
For the present analysis we divided the cohort into three distinct groups based on breastfeeding exposure: Participants who did not breastfeed (none), participants with less than 6 months exposure to breastfeeding ( $<6$ months), and participants with 6 months or more exposure to breastfeeding ( $\geq 6$ months). Other sources of feeding (i.e., formula, solids), reasons for not breastfeeding, and reasons for discontinuation before 6 months postpartum were not routinely recorded.

\section{Outcome data}

Seated systolic and diastolic blood pressure (SBP and DBP, respectively) were recorded by the research nurse for all participants at the clinic visit using the standard technique. $^{22}$ Following a minimum of 10 minutes at rest in a sitting position, a total of six BP measures were taken using an automated BP machine; the first measure was excluded, and an average of the remaining measures was recorded. This method has been shown to improve the accuracy of measurements and correlate well with 24-hour ambulatory BP results, and is the preferred measurement recommended by Hypertension Canada. $^{22-25}$ SBP was specified as elevated $(120-129 \mathrm{mmHg})$ or hypertensive $(\geq 130 \mathrm{mmHg})$, and DBP as hypertensive $(\geq 80 \mathrm{mmHg}){ }^{26}$ Height, weight, and abdominal circumference were measured and body mass index was calculated on all women (weight $[\mathrm{kg}] /$ height $\left[\mathrm{m}^{2}\right]$ ). We categorized BMI as low $\left(<18.5 \mathrm{~kg} / \mathrm{m}^{2}\right)$, normal $\left(18.5-25 \mathrm{~kg} / \mathrm{m}^{2}\right)$, overweight $\left(25-30 \mathrm{~kg} / \mathrm{m}^{2}\right)$, and obese $\left(\geq 30 \mathrm{~kg} / \mathrm{m}^{2}\right)$ as per World Health Organization categories. $^{27}$

Biochemical measurements included serum total cholesterol, high-density lipoprotein-cholesterol (HDL-C), low-density lipoprotein-cholesterol (LDL-C), triglycerides, fasting glucose, ratio of total cholesterol to HDL-C, and non-HDL-C (referred to herein as the "metabolic panel"). Data collected at the MHC visit were used to calculate the Lifetime Risk of CVD score (the cumulative risk assessment of developing CVD in one's lifetime $^{28}$ ) and to determine the presence of metabolic syndrome. The Lifetime Risk Score is determined based on cholesterol, $\mathrm{BP}$, smoking, and fasting glucose, with scores stratified as high $(\geq 39 \%)$ or low risk $(<39 \%){ }^{28}$ This scoring system has shown better utility in young female cohorts, as compared with other risk scores such as the Framingham Risk Score. ${ }^{29}$ Metabolic syndrome was defined by the presence of three or more of (1) abdominal obesity with waist circumference $>88 \mathrm{~cm}$ in women, (2) triglyceride level $\geq 150 \mathrm{mg} / \mathrm{dL}$, (3) $\mathrm{HDL}-\mathrm{C}<50 \mathrm{mg} / \mathrm{dL}$ in women, (4) $\mathrm{SBP} \geq 130 \mathrm{mmHg}$ or $\mathrm{DBP} \geq 85 \mathrm{mmHg}$, or (5) fasting glucose $\geq 100 \mathrm{mg} / \mathrm{dL}^{30}$

\section{Covariate data}

Sociodemographic data collected at the MHC include ethnicity, highest level of education achieved (grade or high school, postsecondary school not completed, postsecondary school completed), household income $(<\$ 29,000, \$ 30,000$ $\$ 59,000, \$ 60,000-\$ 89,000$, and $\geq \$ 90,000)$, tobacco use (any or none), and level of physical activity (days/week). Prepregnancy weight and height was abstracted from the patient's chart. Prepregnancy BMI and gestational weight gain was determined based on this value. This information was verified with the patient at their visit and missing values were filled in based on self-report. Days physically active was defined as self-reported days per week where the participant completed 30 minutes or more of moderate physical activity. 
A list of pre-existing medical conditions, family history of medical conditions, prior pregnancies, births, and use of assisted reproduction were also recorded.

\section{Statistical analyses}

Descriptive analyses were performed and compared in three groups based on exposure to breastfeeding: participants who did not breastfeed (none), participants with less than 6 months exposure to breastfeeding ( $<6$ months), and participants with at least 6 months exposure to breastfeeding ( $\geq 6$ months). The Kruskal-Wallis test was used to compare continuous variables and the chi squared test for categorical variables between groups. All statistical tests were performed using two-sided tests and a family-wise level of significance of 0.05 . The Bonferroni method was used to adjust for the family-wise error rate. The overall $p$-value of 0.05 was divided by three and $p<0.0167$ was used to determine significance for each pairwise comparison. $p$-values are reported to three decimal places with values less than 0.001 reported as $<0.001$.

Multivariate analyses were performed using linear, logistic, or multinomial regression to examine the association between breastfeeding exposure and calculated CVD risk scores, presence of metabolic syndrome, physical measures (e.g., $\mathrm{BP}$ and $\mathrm{BMI})$, and individual elements of the metabolic

Table 1. Participant Characteristics at the Maternal Health Clinic Appointment BY DURATION OF BREASTFEEDING

\begin{tabular}{|c|c|c|c|c|}
\hline Characteristic & None $(\mathrm{N}=100)$ & $<6$ Months $(\mathrm{N}=315)$ & $\geq 6$ Months $(\mathrm{N}=207)$ & $\mathrm{p}$ \\
\hline Age (years), median (IQR) & $32(29-36)$ & $31(27-35)$ & $32(29-36)$ & $0.028 \dagger$ \\
\hline Prepregnancy BMI $\left(\mathrm{kg} / \mathrm{m}^{2}\right)$, median (IQR) & $28.6(24.4-34.8)^{\mathrm{b}}$ & $26.2(22.4-32.0)^{\mathrm{b}}$ & $25.5(22.6-31.2)$ & $0.004 * \dagger$ \\
\hline Overweight $25.0-29.9, n(\%)$ & $27(27.6)$ & $71(22.7)$ & $57(27.5)$ & $0.012 *^{*}$ \\
\hline Obese $\geq 30.0, n(\%)$ & $45(45.9)$ & $103(32.9)$ & $58(28.0)$ & \\
\hline \multicolumn{5}{|l|}{ Race, $n(\%)$} \\
\hline Caucasian & $92(92.9)^{\mathrm{a}}$ & $270(86.5)^{\mathrm{c}}$ & $170(83.3)^{\mathrm{c}}$ & \multirow[t]{2}{*}{0.072} \\
\hline Other & $7(7.1)$ & $42(13.5)$ & $34(16.7)$ & \\
\hline \multicolumn{5}{|l|}{ Highest education level completed $^{\alpha}, n(\%)$} \\
\hline Postsecondary completed & $67(68.4)^{\mathrm{b}}$ & $252(81.3)^{\mathrm{d}}$ & $184(89.3)^{\mathrm{a}}$ & \multirow[t]{3}{*}{$<0.001^{*}, \dagger$} \\
\hline Postsecondary not complete & $10(10.2)$ & $15(4.8)$ & $12(5.8)$ & \\
\hline Grade or high school & $21(21.4)$ & 43 (13.9) & $10(4.9)$ & \\
\hline \multicolumn{5}{|l|}{ Household income, $n(\%)$} \\
\hline$\leq \$ 29,000$ & $14(15.4)^{\mathrm{e}}$ & $32(10.7)^{\mathrm{g}}$ & $8(4.2)^{\mathrm{f}}$ & \multirow[t]{4}{*}{$<0.001 * t$} \\
\hline$\$ 30,000-\$ 59,000$ & $31(34.4)$ & $64(21.5)$ & $32(16.8)$ & \\
\hline$\$ 60,000-\$ 89,000$ & $28(31.1)$ & $82(27.5)$ & $55(28.8)$ & \\
\hline$\geq \$ 90,000$ & $17(18.9)$ & $120(40.3)$ & $96(50.3)$ & \\
\hline Smoking, $n(\%)$ & $19(19.0)$ & $37(11.7)$ & $10(4.8)$ & $<0.001 * \dagger$ \\
\hline Pre-existing diabetes, $n(\%)$ & $1(1.0)$ & $3(1.0)$ & $1(0.5)$ & 0.82 \\
\hline \multicolumn{5}{|l|}{ Days physically active per week ${ }^{\beta}, n(\%)$} \\
\hline 0 & $53(53.0)$ & $116(37.4)^{\mathrm{d}}$ & $74(35.7)$ & \multirow[t]{4}{*}{$0.014 *+$} \\
\hline $1-3$ & $19(19.0)$ & $95(30.6)$ & $70(33.8)$ & \\
\hline $4-5$ & $13(13.0)$ & $68(21.9)$ & 37 (17.9) & \\
\hline $6-7$ & $15(15.0)$ & $31(10.0)$ & $26(12.6)$ & \\
\hline \multicolumn{5}{|l|}{ Parity, $n(\%)$} \\
\hline 1 & $35(35.0)$ & $167(53.2)^{\mathrm{a}}$ & $86(41.7)^{\mathrm{a}}$ & \multirow[t]{3}{*}{$0.009 *, \$$} \\
\hline 2 & $30(30.0)$ & $65(20.7)$ & $54(26.2)$ & \\
\hline$\geq 3$ & $35(35.0)$ & $80(25.5)$ & $66(32.0)$ & \\
\hline Gestational weight gain $(\mathrm{kg})$, median (IQR) & $13.0(8.4-21.1)^{\mathrm{h}}$ & $15.0(10.0-20.6)^{\mathrm{i}}$ & $14.0(9.0-20.0)^{\mathrm{j}}$ & 0.49 \\
\hline Excess gestational weight gain ${ }^{\gamma}, n(\%)$ & $53(57.6)$ & $173(59.5)$ & $108(55.4)$ & 0.82 \\
\hline \multicolumn{5}{|l|}{ History of pregnancy complications $\delta, n(\%)$} \\
\hline Gestational diabetes & $27(27.0)$ & $81(25.7)$ & $63(30.4)$ & 0.49 \\
\hline Hypertensive disorder & $55(55.0)$ & $173(54.9)$ & $97(46.9)$ & 0.16 \\
\hline Intrauterine growth restriction & $12(12.0)$ & $27(8.6)$ & $19(9.2)$ & 0.59 \\
\hline Abruption & $6(6.0)$ & $15(4.8)$ & $7(3.4)$ & 0.56 \\
\hline Preterm birth & $11(11.0)$ & 47 (14.9) & $24(11.6)$ & 0.43 \\
\hline
\end{tabular}

Table information: ${ }^{\alpha}$ Postsecondary completed indicates that a degree or diploma has been completed, postsecondary not complete indicates that some classes were taken but a degree or diploma was not received, grade or high school completed categories were grouped into one category because of small cell counts; ${ }^{\beta}$ Self-reported days per week the participant completed 30 minutes or more of moderate physical activity; ' Gestational weight gain determined based on ppBMI: ppBMI $<18.5$ and $>18.0 \mathrm{~kg}$ gained, ppBMI $18.5-24.9$ and $>16.0 \mathrm{~kg}$ gained, ppBMI $25.0-29.9$ and $>11.5 \mathrm{~kg}$ gained, and ppBMI $>29.9$ and $>9.0 \mathrm{~kg}$ gained ${ }^{47} ;{ }^{\delta}$ Complications from any previous pregnancy, a participant may have experienced more than one type of complication, if the participant experienced a given complication in multiple pregnancies it is only counted once. $\boldsymbol{p}$-Values: The $p$-values presented are for the overall comparisons of the three groups by KruskalWallis or chi square test. Further information on the corrected pairwise comparisons are provided by the asterisks. Pairwise comparisons: $* \geq 6$ Months versus none; ${ }^{\dagger} \geq 6$ Months versus $<6$ months; ${ }^{t}<6$ Months versus none; For three pairwise comparisons at $p=0.05 / 3=0.0167$.

Missing values: a: 1; b: 2; c: 3; d: 5; e: 10; f: 16; g: 17; h: 7; i: 24; j: 12 .

BMI, body mass index; IQR, interquartile range; ppBMI, prepregnancy body mass index. 


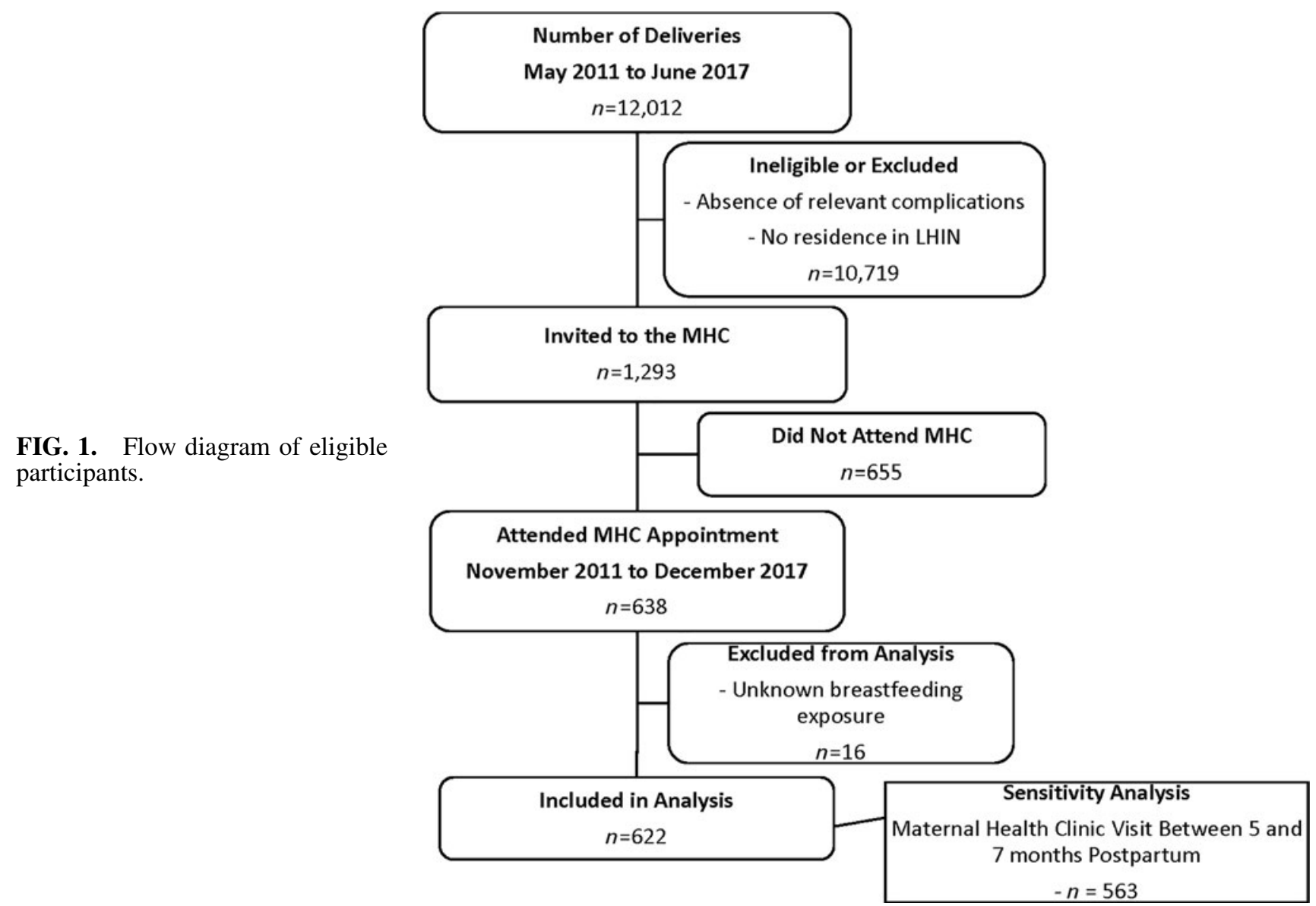

panel (e.g., high-density lipoprotein [HDL], low-density lipoprotein and glucose). For these models, breastfeeding exposure was considered as a continuous variable measured in months. For all models the following were considered as confounding variables: age, ethnicity, education, income, smoking, parity, physical activity, time postpartum, prepregnancy BMI, and gestational weight gain. The categorical variable levels are outlined in Table 1. Multivariate analyses were also performed, where breastfeeding exposure was considered a binary variable using "no breastfeeding" as the referent group. Unadjusted and adjusted models are included as Supplementary Tables S1-S4 (See Appendix).

A sensitivity analysis was performed for participants who visited the MHC between 5 and 7 months to ensure that all individuals had an equal opportunity to breastfeed for $\sim 6$ months. We thus excluded women who visited the $\mathrm{MHC}$ at $<5$ or $\geq 8$ months ( 9 women from the "None" group, 29 from the " $<6$ Months" group, and 21 women from the " $\geq 6$ Months" group), resulting in a sample size of 563 women included in this analysis. Similar multivariate analyses using linear, logistic, or multinomial regression models were performed for this subgroup. All analyses were performed using SPSS Statistics version 24 (SPSS, Inc., Chicago, IL).

\section{Results}

Between November 2011 and December 2017, 638 women attended the MHC for a pregnancy complication (Fig. 1).
After excluding 16 women with unknown breastfeeding status at their MHC visit, 622 women were included in the analysis. Of these women, $325(49 \%)$ had HDP, $171(26 \%)$ had GDM, $58(9 \%)$ had IUGR, $28(4 \%)$ had abruption, and 82 (12\%) had preterm birth, and these complications were not mutually exclusive. The median time postpartum at the time of the MHC visit was 6.1 months [interquartile range 5.7-6.7] with a range of 1.8-14.9 months. The women were analyzed by three groups based on breastfeeding exposure: 207 (33\%) women breastfed for $\geq 6$ months, $315(51 \%)$ breastfed for less than 6 months, and $100(16 \%)$ did not breastfeed.

Baseline characteristics are presented in Table 1. Women who breastfed $\geq 6$ months reported a higher level of education completed compared with those who breastfed $<6$ months, and compared with those who did not breastfeed. Household income, physical activity, and parity were also significantly higher in breastfeeding women ( $<6$ and $\geq 6$ months) compared with women who did not breastfeed. There were no betweengroup differences in race, pre-existing diabetes, or a prior history of pregnancy complications.

Table 2 presents CVD risk factors according to category of breastfeeding duration. All the following pairwise comparisons are statistically significant at the adjusted $p$-value $<0.0167$. Women who breastfed for $\geq 6$ months had significantly lower triglycerides, fasting serum glucose, ratio of cholesterol to HDL-C and higher HDL-C. BMI, waist circumference, and SBP were also significantly lower in women who were still breastfeeding at 6 months. The 
Table 2. Biochemical Measurements, Physical Measurements, and Risk Assessments at the Time of Maternal Health Clinic Visit by Duration of Breastfeeding

\begin{tabular}{|c|c|c|c|c|}
\hline Biochemical measurements & None $(\mathrm{N}=100)$ & $\begin{array}{l}<6 \text { Months } \\
(\mathrm{N}=315)\end{array}$ & $\begin{array}{l}\geq 6 \text { Months } \\
(\mathrm{N}=207)\end{array}$ & $\mathrm{p}$ \\
\hline $\begin{array}{l}\text { Total cholesterol }(\mathrm{mmol} / \mathrm{L}) \text {, median }(\mathrm{IQR}) \\
\geq 5.16(\mathrm{mmol} / \mathrm{L}), n(\%)\end{array}$ & $\begin{array}{l}\mathbf{4 . 6 1}\left(\mathbf{( 4 . 0 4 - 5 . 3 1 ) ^ { \mathrm { a } }}\right. \\
\quad 21(27.3)\end{array}$ & $\begin{array}{l}\mathbf{4 . 6 2}(\mathbf{4 . 0 9 - 5 . 2 1})^{\mathrm{c}} \\
\quad 70(27.3)\end{array}$ & $\begin{array}{l}4.48(3.96-5.04)^{\mathrm{g}} \\
35(20.7)\end{array}$ & $\begin{array}{l}\mathbf{0 . 2 2} \\
0.27\end{array}$ \\
\hline $\begin{array}{l}\text { HDL-C }(\mathrm{mmol} / \mathrm{L}), \text { median }(\mathrm{IQR}) \\
\quad<0.90(\mathrm{mmol} / \mathrm{L}), n(\%)\end{array}$ & $\begin{array}{l}1.24(1.09-1.51)^{\mathrm{a}} \\
\quad 5(6.5)\end{array}$ & $\begin{array}{l}1.39(1.20-1.62)^{d} \\
\quad 9(3.5)\end{array}$ & $\begin{aligned} 1.42 & \left(1.23-1.67^{\mathrm{g}}\right. \\
5 & (3.0)\end{aligned}$ & $\begin{array}{l}<0.001 * \dagger \\
0.38\end{array}$ \\
\hline $\begin{array}{l}\text { LDL-C }(\mathrm{mmol} / \mathrm{L}), \text { median }(\mathrm{IQR}) \\
\quad \geq 3.50(\mathrm{mmol} / \mathrm{L}), n(\%)\end{array}$ & $\begin{array}{l}\mathbf{2 . 8 3}(\mathbf{2 . 2 1 - 3 . 2 7})^{\mathbf{b}} \\
13(17.3)\end{array}$ & $\begin{array}{l}2.63(\mathbf{2 . 2 1}-\mathbf{3 . 1 8})^{\mathrm{e}} \\
\quad 43(16.9)\end{array}$ & $\begin{array}{l}\mathbf{2 . 6 2}(\mathbf{2 . 1 5 - 2 . 9 9 )})^{\mathbf{i}} \\
22(13.2)\end{array}$ & $\begin{array}{l}\mathbf{0 . 3 6} \\
0.54\end{array}$ \\
\hline $\begin{array}{l}\text { Triglycerides }(\mathrm{mmol} / \mathrm{L}), \text { median }(\mathrm{IQR}) \\
\geq 1.70(\mathrm{mmol} / \mathrm{L}), n(\%)\end{array}$ & 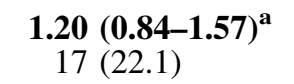 & $\begin{array}{l}\mathbf{0 . 9 5}(\mathbf{0 . 6 6 - 1 . 5 1})^{\mathbf{c}} \\
44(17.2)\end{array}$ & $\begin{array}{l}\mathbf{0 . 7 3}(\mathbf{0 . 5 5 - 1 . 0 5})^{\mathbf{g}} \\
22(13.0)\end{array}$ & $\begin{array}{l}<\mathbf{0 . 0 0 1} * \dagger \dagger \\
0.19\end{array}$ \\
\hline $\begin{array}{l}\text { Fasting glucose }(\mathrm{mmol} / \mathrm{L}), \text { median }(\mathrm{IQR}) \\
>5.6(\mathrm{mmol} / \mathrm{L}), n(\%)\end{array}$ & $\begin{array}{l}4.9(4.5-5.2)^{b} \\
\quad 6(8.0)\end{array}$ & $\begin{array}{l}4.7(4.4-5.0)^{\mathbf{f}} \\
14(5.6)\end{array}$ & $\begin{array}{l}\mathbf{4 . 6}(\mathbf{4 . 4 - 4 . 9})^{\mathrm{h}} \\
\quad 2(1.2)\end{array}$ & $\begin{array}{c}<\mathbf{0 . 0 0 1} * \dagger \\
0.028 *\end{array}$ \\
\hline $\begin{array}{l}\text { Ratio total cholesterol:HDL-C, median } \\
\text { (IQR) }\end{array}$ & $3.7(3.0-4.3)^{\mathrm{a}}$ & $3.2(2.7-4.0)^{\mathrm{d}}$ & $3.2(2.6-3.8)^{\mathrm{g}}$ & $<0.001 * \dagger$ \\
\hline $\begin{array}{l}\geq 3.5, n(\%) \\
\text { Non-HDL-C }(\mathrm{mmol} / \mathrm{L}), \text { median }(\mathrm{IOR})\end{array}$ & $\begin{array}{l}49(63.6) \\
\mathbf{3 . 3 6}(\mathbf{2 . 7 8 - 4 . 1 0})^{\mathbf{a}}\end{array}$ & $\begin{aligned} & 98(38.4) \\
& \mathbf{3 . 1 2}(\mathbf{2 . 6 1 - 3 . 7 4})^{\mathbf{d}}\end{aligned}$ & $\begin{aligned} 62(36.7) \\
\mathbf{2 . 9 9}(\mathbf{2 . 5 1 - 3 . 6 4})^{\mathrm{g}}\end{aligned}$ & $<0.001 * \dagger$ \\
\hline$\geq 4.3(\mathrm{mmol} / \mathrm{L}), n(\%)$ & $12(15.6)$ & $33(12.9)$ & & \\
\hline $\begin{array}{l}\text { Physical measurements } \\
\text { SBP }(\mathrm{mmHg}), \text { median }(\mathrm{IQR}) \\
\text { Elevated 120-129(mmg), } n(\%) \\
\text { Hypertensive } \geq 130(\mathrm{mmHg})^{\alpha}, n(\%)\end{array}$ & $\begin{aligned} 120 & (\mathbf{1 1 1}-\mathbf{1 3 0})^{\mathbf{j}} \\
25 & (25.3) \\
28 & (28.3)\end{aligned}$ & $\begin{array}{l}117(\mathbf{1 1 1 - 1 2 7})^{\mathbf{k}} \\
72(23.1) \\
58(18.6)\end{array}$ & $\begin{array}{l}114(\mathbf{1 0 8}-\mathbf{1 2 2}) \\
41(19.8) \\
27(13.0)\end{array}$ & $\begin{array}{l}\mathbf{0 . 0 0 1 *}{ }^{*} \dagger \\
0.006^{*}\end{array}$ \\
\hline $\begin{array}{l}\text { DBP }(\mathrm{mmHg}) \text {, median }(\mathrm{IQR}) \\
\quad \text { Hypertensive } \geq 80(\mathrm{mmHg})^{\alpha}, n(\%)\end{array}$ & $\begin{array}{l}81(73-90)^{\mathbf{j}} \\
55(55.6)\end{array}$ & $\begin{array}{l}\mathbf{8 0}(\mathbf{7 4 - 8 7})^{\mathbf{k}} \\
172(55.1)\end{array}$ & $\begin{array}{l}\mathbf{7 8}(\mathbf{7 3 - 8 5}) \\
94(45.4)\end{array}$ & $\begin{array}{l}\mathbf{0 . 0 6 7} \\
0.07\end{array}$ \\
\hline $\begin{array}{l}\text { Antihypertensive use, } n(\%) \\
\text { BMI }\left(\mathrm{kg} / \mathrm{m}^{2}\right) \text {, median (IQR) } \\
\text { Overweight } 25.0-29.9\left(\mathrm{~kg} / \mathrm{m}^{2}\right), n(\%) \\
\text { Obese } \geq 30.0\left(\mathrm{~kg} / \mathrm{m}^{2}\right), n(\%)\end{array}$ & $\begin{array}{l}\mathbf{5}(\mathbf{5 . 0}) \\
\mathbf{3 2 . 5}(\mathbf{2 6 . 8 - 3 9 . 6})^{\mathbf{j}} \\
28(28.3) \\
57(57.6)\end{array}$ & $\begin{aligned} \mathbf{6} & (\mathbf{1 . 9}) \\
\mathbf{2 8 . 4} & (\mathbf{2 4 . 8 - 3 4 . 9 )} \\
93 & (29.5) \\
134 & (42.5)\end{aligned}$ & $\begin{aligned} & 5(\mathbf{2 . 4}) \\
\mathbf{2 7 . 1} & (\mathbf{2 3 . 7 - 3 3 . 7 )} \\
57 & (27.5) \\
79 & (38.2)\end{aligned}$ & $\begin{aligned} \mathbf{0 . 2 3} \\
<\mathbf{0 . 0 0 1} * \dagger \\
0.003 *, \$\end{aligned}$ \\
\hline $\begin{array}{l}\text { Waist circumference }(\mathrm{cm}), \text { median (IQR) } \\
\quad 80.0-88.0(\mathrm{~cm}), n(\%) \\
>88.0(\mathrm{~cm}), n(\%)\end{array}$ & $\begin{array}{l}\mathbf{1 0 0 . 6}(\mathbf{8 9 . 3 - 1 1 6 . 5})^{\mathbf{j}} \\
12(12.1) \\
76(76.8)\end{array}$ & $\begin{array}{l}\text { 93.0 (83.7-109.4) } \\
62(19.7) \\
197(62.7)\end{array}$ & $\begin{array}{l}\mathbf{9 0 . 0}(\mathbf{8 2 . 0 - 1 0 4 . 3})^{\mathbf{j}} \\
49(23.8) \\
115(55.8)\end{array}$ & $\begin{array}{r}<0.001 *, \$ \\
0.014 *, \$\end{array}$ \\
\hline $\begin{array}{l}\text { Risk assessments } \\
\text { High lifetime risk of } \mathrm{CVD}^{\beta}, n(\%) \\
\text { Metabolic syndrome criteria } \mathrm{Met}^{\gamma}, n(\%)\end{array}$ & $\begin{array}{l}48(63.2)^{1} \\
22(29.3)^{b}\end{array}$ & $\begin{aligned} 115(45.1)^{\mathrm{d}} \\
52(21.0)^{\mathrm{m}}\end{aligned}$ & $\begin{array}{l}67(39.9)^{\mathrm{h}} \\
26(15.7)^{\mathrm{n}}\end{array}$ & $\begin{array}{l}\text { 0.003*, } \\
\text { 0.049* }\end{array}$ \\
\hline
\end{tabular}

Table Information: ${ }^{\alpha}$ Or using an antihypertensive; ${ }^{\beta}$ The Lifetime risk score (the cumulative risk assessment of developing CVD in one's lifetime) is determined based on cholesterol, BP, smoking, and fasting glucose, with scores defined as high when $\geq 39 \%{ }^{28}$; ${ }^{\gamma}$ Metabolic syndrome was defined by the presence of three or more of (1) abdominal obesity with waist circumference $>88 \mathrm{~cm}$ in women, (2) triglyceride level $\geq 150 \mathrm{mg} / \mathrm{dL}$, (3) HDL-C $<50 \mathrm{mg} / \mathrm{dL}$ in women, (4) $\mathrm{SBP} \geq 130 \mathrm{mmHg}$ or DBP $\geq 85 \mathrm{mmHg}$, or (5) fasting glucose $\geq 100 \mathrm{mg} / \mathrm{dL} .{ }^{30} \boldsymbol{p}$-Values: The $p$-values presented are for the overall comparisons of the three groups by Kruskal-Wallis or chi square test. Further information on the corrected pairwise comparisons are provided by the asterisks. Pairwise comparisons: $* \geq 6$ Months versus none; ${ }^{+} \geq 6$ Months versus $<6$ Months; ${ }^{*}<6$ Months versus none; for three pairwise comparisons at $p=0.05 / 3=0.0167$. Missing values: a: 23 ; b: 25 ; c: 59 ; d: 60; e: 61 ; f: 64; g: 38; h: 39; i: 40; j: 1; k: 3; 1: 24; m: 67; n: 41.

Values in bold indicate statistical significance.

BP, blood pressure; DBP, diastolic blood pressure; HDL-C, high-density lipoprotein-cholesterol; LDL-C, low-density lipoproteincholesterol; SBP, systolic blood pressure.

proportion of women with a high lifetime risk of CVD was significantly lower among women with any breastfeeding compared with those who did not breastfeed. The percentage of women with metabolic syndrome was lower in women who breastfed $\geq 6$ months compared with women who did not breastfeed.

The effect of increasing breastfeeding duration in months (up to 6 months) on the odds of abnormal risk scores, physical measures, and blood work, before and after adjusting for confounding variables with logistic or multinomial regression, can be found in Table 3 . The following outcomes were found to have adjusted odds ratios for breastfeeding duration in months that were significant at $p<0.05$. The odds of having metabolic syndrome were statistically significantly decreased with increasing breastfeeding duration. The odds of having abnormal fasting glucose or ratio of total cholesterol to HDL-C also decreased significantly.

The effect of increasing breastfeeding duration in months on physical measures and blood work, before and after adjusting for confounding variables with linear regression, can be found in Table 4. BMI, fasting glucose, triglycerides, and the ratio of total cholesterol to HDL-C significantly decreased with increasing breastfeeding duration. HDL significantly increased with increasing breastfeeding duration.

The sensitivity analyses include women who visited the MHC within 5-7 months postpartum $(N=563)$ (see Supplementary Tables S5-S8 in Supplementary Appendix SA1). Increased breastfeeding duration was associated with lower physical and chemical markers for $\mathrm{CV}$ risk. The proportion of 
Table 3. Odds Ratio Estimates for Duration of Breastfeeding (Months) - Logistic OR Multinomial Regression

\begin{tabular}{|c|c|c|c|c|c|c|}
\hline Outcome for the model & $\mathrm{N}$ & Unadjusted OR (95\% CI) & $\mathrm{p}$ & $\mathrm{N}$ & Adjusted $O R^{\alpha}(95 \% C I)$ & $\mathrm{p}$ \\
\hline \multicolumn{7}{|l|}{ Risk scores } \\
\hline Metabolic syndrome ${ }^{\beta}$ & 489 & $0.83(0.76-0.91)$ & $<0.001$ & 415 & $0.89(0.79-0.99)$ & 0.035 \\
\hline High lifetime risk score $\gamma$ & 499 & $0.87(0.81-0.94)$ & $<0.001$ & 423 & $0.95(0.86-1.04)$ & 0.25 \\
\hline \multicolumn{7}{|l|}{ Physical measures } \\
\hline \multicolumn{7}{|l|}{ BMI $\left(\mathrm{kg} / \mathrm{m}^{2}\right)$} \\
\hline Overweight $25.0-29.9$ vs. norma & 621 & $0.90(0.82-0.98)$ & 0.012 & 523 & $0.89(0.74-1.06)$ & 0.20 \\
\hline Obese $\geq 30.0$ vs. normal $<2$ & & $0.85(0.78-0.92)$ & $<0.001$ & & $-1.04)$ & 0.10 \\
\hline \multicolumn{7}{|l|}{ Waist circumference $(\mathrm{cm})$} \\
\hline $80.0-88.0$ vs. Normal $<80.0$ & 619 & $0.99(0.89-1.10)$ & 0.83 & 520 & $1.01(0.85-1.21)$ & 0.89 \\
\hline \multirow{2}{*}{\multicolumn{7}{|c|}{$\mathrm{SBP}(\mathrm{mmHg})$}} \\
\hline & & & & & & \\
\hline Elevated $120-129$ vs. nor & 618 & $0.91(0.84-0.99)$ & $\mathbf{0 . 0 2 2}$ & 520 & $0.91(0.83-1.00)$ & 0.051 \\
\hline Hypertensive $\geq 130$ vs. normal $<120$ & & $0.85(0.78-0.92)$ & $<0.001$ & & $0.94(0.84-1.04)$ & 0.22 \\
\hline \multicolumn{7}{|l|}{ DBP $(\mathrm{mmHg})$} \\
\hline Hypertensive $\geq 80$ vs. normal $(<80)$ & 618 & $0.93(0.87-0.99)$ & 0.022 & 520 & $0.94(0.87-1.02)$ & 0.16 \\
\hline \multicolumn{7}{|l|}{ Blood work } \\
\hline Total cholesterol $\geq 5.16 \mathrm{mmol} / \mathrm{L}$ & 502 & $0.95(0.88-1.03)$ & 0.20 & 425 & $0.96(0.86-1.06)$ & 0.42 \\
\hline $\mathrm{HDL}-\mathrm{C}<0.09 \mathrm{mmol} / \mathrm{L}$ & 501 & $0.82(0.68-0.98)$ & 0.03 & 425 & $0.92(0.74-1.14)$ & 0.46 \\
\hline $\mathrm{LDL}-\mathrm{C} \geq 3.50 \mathrm{mmol} / \mathrm{L}$ & 496 & $0.93(0.84-1.02)$ & 0.13 & 421 & $0.97(0.85-1.09)$ & 0.59 \\
\hline Fasting glucos & 494 & $0.78(0.66-0.93)$ & 0.005 & 419 & $0.79(0.64-0.96)$ & 0.019 \\
\hline Triglycerides $\geq 1.70 \mathrm{mmol} / \mathrm{L}$ & 502 & $0.85(0.77-0.93)$ & 0.001 & 425 & $0.89(0.79-1.01)$ & 0.066 \\
\hline Total cholesterol:HDL-C $\geq 3.0$ & 501 & $0.84(0.78-0.90)$ & $<0.001$ & 425 & $0.86(0.78-0.95)$ & 0.003 \\
\hline Non-HDL-C $\geq 4.30 \mathrm{mmol} / \mathrm{L}$ & 501 & $0.90(0.81-0.99)$ & 0.047 & 425 & $0.94(0.82-1.07)$ & 0.33 \\
\hline
\end{tabular}

${ }^{\alpha}$ Adjusted for age (years), race, education, income, smoking, parity, physical activity (days/week), time postpartum (months), ppBMI $\left(\mathrm{kg} / \mathrm{m}^{2}\right)$, and gestational weight gain $(\mathrm{kg})$. Groupings for the categorical variables as described in Table 1; ${ }^{\beta}$ Metabolic syndrome was defined by the presence of three or more of (1) abdominal obesity with waist circumference $>88 \mathrm{~cm}$ in women, (2) triglyceride level $\geq 150 \mathrm{mg} / \mathrm{dL}$, (3) HDL-C $<50 \mathrm{mg} / \mathrm{dL}$ in women, (4) SBP $\geq 130 \mathrm{mmHg}$ or DBP $\geq 85 \mathrm{mmHg}$, or (5) fasting glucose $\geq 100 \mathrm{mg} / \mathrm{dL}^{30}$; ${ }^{\gamma} \mathrm{The}$ Lifetime risk score (the cumulative risk assessment of developing CVD in one's lifetime) is determined based on cholesterol, BP, smoking and fasting glucose, with scores defined as high when $\geq 39 \%$. ${ }^{28}$

Values in bold indicate statistical significance.

CI, confidence interval; CVD, cardiovascular disease; OR, odds ratio.

women with a high lifetime risk of CVD and metabolic syndrome was associated with increased breastfeeding.

\section{Discussion}

This study found that in a cohort of postpartum women with a pregnancy complication associated with an increased risk of future CVD, there seemed to be a dose-response effect of increased breastfeeding on various biochemical, physical, and metabolic markers of CVD risk measured at 6 months postpartum. The results of our study are mostly congruent with previous research investigating the effects of breastfeeding duration and various CVD markers. In a meta-analysis of 21 retrospective and prospective studies, breastfeeding was associated with lower odds of metabolic risk factors, hypertension, type 2 diabetes, and prevalent CVD in the general population. The same review reported little or no association between breastfeeding and postpartum weight change and body composition. ${ }^{12}$ However, our cohort differs from previous published work in the inclusion of women with excess risk of future CVD based on a recent complicated pregnancy.

Evidence of the effects of breastfeeding specifically in women with HDP is still scarce and inconclusive. One other study of 379 women found a reduction in BP in women with a history of GH when comparing those who breastfed for greater than 6 months to those who did not breastfeed at all. ${ }^{17}$ Another cohort study of 1,238 women in the United States showed an inverse association between breastfeeding duration and diabetes incidence. ${ }^{18} \mathrm{~A}$ recent study by Countouris et al. found that breastfeeding duration among women with $\mathrm{GH}$ and preeclampsia was not associated with specific measures of inflammation and renal function. ${ }^{31}$

Overall, the results from our study build on the existing body of literature suggesting that breastfeeding is associated with potential improvements of short-term cardiometabolic health outcomes, and that these benefits may be further augmented among women who have had a pregnancy complication. Although CVD risk is a long-term matter and we can presently only speculate on any future benefits of breastfeeding, our results demonstrate the importance of early identification of these higher risk women to receive primary prevention as opposed to management later in life when there is potentially a greater disease burden on CVD. The addition of supportive breastfeeding interventions as part of an overall CVD risk reduction strategy should be tested and implemented in both the perinatal period and in specialized postpartum CVD risk reduction clinics such as the MHC.

\section{Clinical implications}

While the WHO recommends exclusive breastfeeding for 6 months, ${ }^{32}$ only $26 \%$ of Canadian women follow this guideline and many incur a sharp drop-off in EBF by 6 weeks postpartum due to factors such as perceived poor milk supply, 


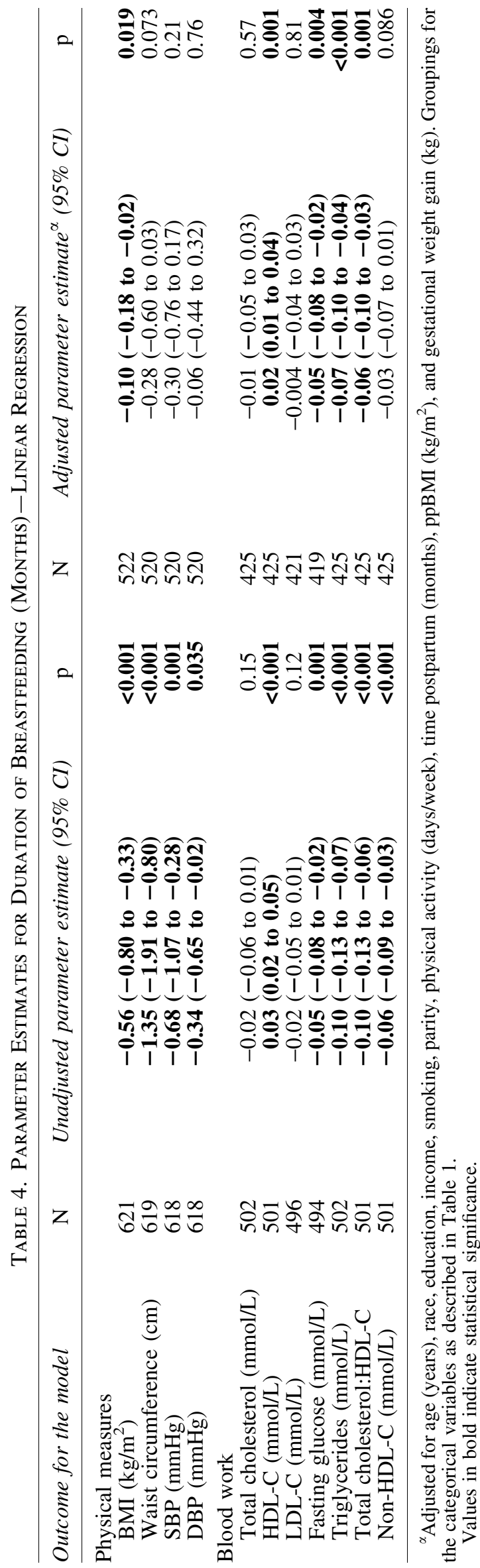

difficulty with breastfeeding techniques, and fatigue. ${ }^{33,34}$ Women with pregnancy complications such as HDP are at even higher risk of early weaning and lower initiation rates of breastfeeding due to specific biological (endothelial dysfunction, metabolic syndrome, obesity), psychosocial (depression, anxiety), and contextual (low birth weight or preterm birth) factors. ${ }^{13-16}$ Our results support the recommendation that breastfeeding at least until 6 months postpartum may provide protective effects against preclinical CVD.

Furthermore, women with pregnancy complications who have difficulty initiating and maintaining breastfeeding should especially be supported through breastfeeding interventions. Enhancing breastfeeding self-efficacy has the potential to overcome the challenges of engaging these women in postpartum maintenance of healthy behaviors other than breastfeeding. ${ }^{35}$ Furthermore, since many women with HDP carry a new diagnosis of hypertension within the first postpartum year and many others have raised BP below hypertension threshold, ${ }^{36,37}$ encouraging successful breastfeeding in the perinatal period may have significant impact in this population. Given the unique vulnerability and challenges of women with pregnancy complications and lack of previous breastfeeding trials in this population, further research should be conducted on the feasibility of perinatal breastfeeding interventions.

\section{Strengths and limitations}

Although randomized controlled trials are the gold standard for determining causal relationships, it is not ethical to randomize women to not breastfeed. Therefore, welldesigned prospective studies that control for potential confounders render the next best evidence. Our study uses data from the largest Canadian cohort of postpartum women with a history of pregnancy complications with robust collection of clinical variables. ${ }^{38}$ As shown in Figure 1, approximately half of the eligible population invited chose to attend the clinic. Previous work has demonstrated that women are more likely to attend if they are older, do not smoke, live closer to the hospital, and live in a Census Metropolitan Area with higher median earnings. ${ }^{38}$ The biases in this clinical population should be considered when generalizing results.

Given the largely Caucasian demographic and higher education and socioeconomic status (SES), the cohort may not be fully representative of the Canadian population. While multivariate modeling has been used in an attempt to account for differences in SES, there may be residual confounding. Furthermore, the relationship among SES, breastfeeding, and CVD risk is complex. This study cannot describe that relationship in entirety and conclusions that are drawn should be limited to the timeframe of the study. Prepregnancy BMI and gestational weight gain were abstracted from the patient's chart and missing values were filled in based on self-report. However, due to the nature of the database, we were unable to ascertain the proportion of these measurements that were self-reported or abstracted from charts.

Due to the fact that breastfeeding status was self-reported, recall or reporting bias may have given rise to misclassification of breastfeeding duration. However, previous research has shown that women with shorter durations of breastfeeding tended to overestimate while women with longer durations tended to underestimate. ${ }^{39}$ This trend would bias our observed effect to the null value and show less of a significant 
difference, suggesting that the effect of breastfeeding might be even more potent. Due to the cross-sectional nature of the measurement, only breastfeeding length in months until the time of the MHC appointment was used in the analysis. Therefore, the measurement used represents exposure to breastfeeding at the time of the CV risk factor assessments. While participants reported the number of months they intended to breastfeed beyond the clinic visit, this was not used in the analysis. Breastfeeding exclusivity or intensity was unknown and the reasons why a woman chose not to breastfeed or decided to stop breastfeeding early were not collected. Breastfeeding and the decision to breastfeed may also be associated with other maternal health behaviors such as physical activity and likelihood of smoking, which were also self-reported. Although many of these demographic factors and lifestyle behaviors were controlled for in the analysis, there may still be residual confounding.

The relationship between breastfeeding and CVD risk indicators may be bidirectional. Women who already have pregnancy complications are likely to have other CVDrelated health issues such as obesity, hypertension, and diabetes, which may exacerbate the challenges associated with initiating or maintaining breastfeeding. Research has found that maternal obesity is associated with discontinuation of breastfeeding and delayed onset of lactogenesis following delivery. ${ }^{40,41}$ This association also gives rise to the causality dilemma of whether breastfeeding prevents metabolic syndrome or if metabolic syndrome prevents breastfeeding. ${ }^{41}$ Observational studies have demonstrated that longer duration of breastfeeding is associated with reduced risk of metabolic disease as lactation is thought to mobilize energy stores in the mother. ${ }^{43,44}$ However, other studies suggest that specific metabolic risk factors such as insulin resistance and elevated BMI are associated with lower breastfeeding rates, suggesting that the direction of the association is inconclusive due to confounding health factors. 45,46

\section{Conclusion}

Our findings show that increased breastfeeding duration is significantly associated with a decreased trend in various short-term markers of CVD risk in a cohort of postpartum women who were diagnosed with a pregnancy complication. Although a causal relationship cannot be definitively established, breastfeeding duration for at least 6 months correlated with potential short-term improvements of direct and indirect measures of maternal CV health. More research is needed to elucidate the physiological basis and duration of the effect of breastfeeding on CVD risk factors. Further investigations are required to determine the ongoing long-term benefits of breastfeeding duration and exclusivity on maternal health.

\section{Author Disclosure Statement}

No competing financial interests exist

\section{Funding Information}

The authors received no specific funding for this work.

\section{Supplementary Material}

Supplementary Appendix SA1

Supplementary Table S1
Supplementary Table S2

Supplementary Table S3

Supplementary Table S4

Supplementary Table S5

Supplementary Table S6

Supplementary Table S7

Supplementary Table S8

\section{References}

1. World Health Organization. Women's Health Fact Sheet No. 334. Geneva: World Health Organization, 2013.

2. Smith GN PJ, Walker M. Ten-year, thirty-year, and lifetime cardiovascular disease risk estimates following a pregnancy complicated by preeclampsia. J Obstet Gynaecol Canada 2012;34:830-835.

3. Fraser A, Nelson SM, Macdonald-Wallis C, et al. Associations of pregnancy complications with calculated cardiovascular disease risk and cardiovascular risk factors in middle age: The Avon Longitudinal Study of Parents and Children. Circulation 2012;125:1367-1380.

4. Smith GN, Walker MC, Liu A, et al. For the Pre-Eclampsia New Emerging Team (PE-NET). A history of preeclampsia identifies women who have underlying cardiovascular risk factors. Am J Obstet Gynecol 2009;200:58e1-58.e8.

5. Stuebe AM, Schwarz EB, Grewen K, et al. Duration of lactation and incidence of maternal hypertension: A longitudinal cohort study. Am J Epidemiol 2013;174:1147-1158.

6. Schwarz EB, Nothnagle M. The maternal health benefits of breastfeeding. Am Fam Phys 2015;91:603-604.

7. Gunderson EP. Impact of breastfeeding on maternal metabolism: Implications for women with gestational diabetes. Curr Diab Rep;14:460.

8. Stuebe AM, Rich-Edwards JW, Willett WC, Manson JE, Michels KB. Duration of lactation and incidence of type 2 diabetes. JAMA 2005;294:2601-2610.

9. McClure CK, Schwarz EB, Conroy MB, Tepper PG, Janssen I, Sutton-Tyrrell KC. Breastfeeding and subsequent maternal visceral adiposity. Obesity 2011;19:2205-2213.

10. Groer MW, Jevitt CM, Sahebzamani F, Beckstead JW, Keefe DL. Breastfeeding status and maternal cardiovascular variables across the postpartum. J Womens Health 2003; 22:453-459.

11. Jonas W, Nissen E, Ransjo-Arvidson AB, Wiklund I, Henriksson P, Uvnas-Moberg K. Short- and long-term decrease of blood pressure in women during breastfeeding. Breastfeed Med 2008;3:103-109.

12. Nguyen B, Jin K, Ding D. Breastfeeding and maternal cardiovascular risk factors and outcomes: A systematic review. PLoS ONE 2017;12:e0187923.

13. Leeners B, Rath W, Kuse S, Neumaier-Wagner P. Breastfeeding in women with hypertensive disorders in pregnancy. J Perinat Med 2005;33:553-560.

14. Cordero L, Valentine CJ, Samuels P, Giannone PJ, Nankervis CA. Breastfeeding in women with severe preeclampsia. Breastfeed Med 2012;7:457-463.

15. Dennis CL, McQueen K. The relationship between infantfeeding outcomes and postpartum depression: A qualitative systematic review. Pediatrics 2009;123:e736-e751.

16. Skalkidou A, Hellgren C, Comasco E, Sylven S, Sundstrom Poroma I. Biological aspects of postpartum depression. Womens Health 2013;8:659-671.

17. Countouris ME, Schwarz EB, Rossiter BC, et al. Effects of lactation on postpartum blood pressure among women with 
gestational hypertension and preeclampsia. Am J Obstet Gynecol 2016;215:241.e1-e8.

18. Gunderson EP, Lewis CE, Lin Y, et al. Lactation duration and progression to diabetes in women across the childbearing years. JAMA Intern Med 2018;178:328-337.

19. Smith GN, Louis JM, Saade G. Pregnancy and the postpartum period as an opportunity for cardiovascular risk identification and management. Obstet Gynecol 2019;134: 851-862.

20. Cusimano MC, Pudwell J, Roddy M, Cho CK, Smith GN. The maternal health clinic: An initiative for cardiovascular risk identification in women with pregnancy-related complications. Am J Obstet Gynecol 2014;210:438.e1-e9.

21. Smith GN. The Maternal Health Clinic: Improving women's cardiovascular health. Semin Perinatol 2015;39:316319.

22. Hypertension Canada. Hypertension Canada Guidelines. Markham, ON: Hypertension Canada, 2018.

23. O'Brien E, Asmar R, Beilin L, et al. European Society of Hypertension recommendations for conventional, ambulatory and home blood pressure measurement. J Hypertens 2003;21:821-848.

24. Brown MA, McHugh L, Mangos G, Davis G. Automated self-initiated blood pressure or 24-hour ambulatory blood pressure monitoring in pregnancy. $\mathrm{Br} \mathrm{J}$ Obstet Gynaecol 2004; 111:38-41.

25. Beckett L, Godwin M. The BpTRU automatic blood pressure monitor compared to 24 hour ambulatory blood pressure monitoring in the assessment of blood pressure in patients with hypertension. BMC Cardiovasc Disord 2005; 5:18.

26. Whelton PK, Carey RM, Aronow WS, et al. 2017 ACC/ AHA/AAPA/ABC/ACPM/AGS/APhA/ASH/ASPC/NMA/ PCNA guideline for the prevention, detection, evaluation, and management of high blood pressure in adults: A report of the American College of Cardiology/American Heart Association Task Force on Clinical Practice Guidelines. J Am Coll Cardiol 2018;71:e127-e248.

27. World Health Organization. WHO BMI Classification. Global database on body mass index. Geneva: World Health Organization, 2006.

28. Lloyd-Jones DM, Leip E, Larson MG, et al. Prediction of lifetime risk for cardiovascular disease by risk factor burden at 50 years of age. Circulation 2006;113:791798.

29. Gladstone RA, Pudwell J, Nerenberg KA, Grover SA, Smith GN. Cardiovascular risk assessment and follow-up of women after hypertensive disorders of pregnancy: A Prospective Cohort Study. J Obstet Gynaecol Can 2019;41: 1157-1167.

30. Kahn R, Buse J, Ferrannini E, Stern M; American Diabetes Association; European Association for the Study of Diabetes. The metabolic syndrome: Time for a critical appraisal: Joint statement from the American Diabetes Association and the European Association for the Study of Diabetes. Diabetes Care 2005;28:2289-2304.

31. Countouris M, Demirci, JR, Jeyabalan A, Catov JM, Schwarz EB. Relationship of postpartum levels of cystatin and high-sensitivity C-reactive protein and duration of lactation in mothers with previous gestational hypertension or preeclampsia. Breastfeed Med 2019;14:408-415.

32. World Health Organization. Global strategy for infant and young child feeding. Geneva: World Health Organization, 2013.
33. Centres for Disease Control \& Prevention. Breastfeeding Report Card. 2016.

34. Brown CR, Dodds L, Legge A, Bryanton J, Semenic S. Factors influencing the reasons why mothers stop breastfeeding. Can J Public Health 2014;105:e179-e185.

35. Brown MC, Best KE, Pearce MS, Waugh J, Robson SC, Bell R. Cardiovascular disease risk in women with preeclampsia: Systematic review and meta-analysis. Eur J Epidemiol 2013;28:1-19.

36. Al-Nasiry S, Ghossein-Doha C, Polman SE, et al. Metabolic syndrome after pregnancies complicated by preeclampsia or small-for-gestational-age: A retrospective cohort. BJOG 2015;122:1818-1823.

37. Smith GC, Pudwell J, Saade GR. Impact of the New American Hypertension Guidelines on the prevalence of postpartum hypertension. Am J Perinatol 2019;36:440-442.

38. Nowik CM, Pudwell J, Smith GN. Evaluating the postpartum maternal health clinic: How patient characteristics predict follow-up. J Obstet Gynaecol Can 2016;38:930935.

39. Promislow JH, Gladen BC, Sandler DP. Maternal recall of breastfeeding duration by elderly women. Am J Epidemiol 2005;161:289-296.

40. Baker JL, Michaelsen K, Sorensen TI, Rasmussen KM. High prepregnant body mass index is associated with early termination of full and any breastfeeding in Danish women. Am J Clin Nutr 2007;86:404-411.

41. Nommsen-Rivers LA, Chantry CJ, Peerson JM, Cohen RJ, Dewey KG. Delayed onset of lactogenesis among first-time mothers is related to maternal obesity and factors associated with ineffective breastfeeding. Am J Clin Nutr 2010; 92:574-584.

42. Stuebe A. Does breastfeeding prevent the metabolic syndrome, or does the metabolic syndrome prevent breastfeeding? Semin Perinatol 2015;39:290-295.

43. Butte NF, Wong WW, Hopkinson JM. Energy requirements of lactating women derived from doubly labeled water and milk energy output. J Nutr 2001;131:53-58.

44. Baker JL, Gamborg M, Heitmann BL, Lissner L, Sorensen TI, Rasmussen KM. Breastfeeding reduces postpartum weight retention. Am J Clin Nutr 2008;88:1543-1551.

45. Matias SL, Dewey KG, Quesenberry CP, Gunderson EP. Maternal prepregnancy obesity and insulin treatment during pregnancy are independently associated with delayed lactogenesis in women with recent gestational diabetes mellitus. Am J Clin Nutr 2014;99:115-121.

46. Lemay DGBO, Hughes MA, Morrow AL, Horseman ND, Nommsen-Rivers LA. RNA sequencing of the human milk fat layer transcriptome reveals distinct gene expression profiles at three stages of lactation. PLoS ONE 2013;8:e67531.

47. Health Canada. Prenatal nutrition guidelines for health professionals: Gestational weight gain. Ottawa, ON: Health Canada. 2014.

Address correspondence to: Graeme N. Smith, MD, PhD, FRCSC Department of Obstetrics and Gynaecology Kingston Health Sciences Centre 76 Stuart Street, Victory 4 Kingston

Ontario K7L 2V7 Canada

E-mail: gns@queensu.ca 